\title{
Growth and structural characterization of molecular superlattice of
}

\section{quaterrylene and $\mathrm{N}, \mathrm{N}$ '-dioctyl-3,4,9,10-perylenedicarboximide}

Nobuya Hiroshiba ${ }^{1,2}$, Ryoma Hayakawa ${ }^{1}$, Matthieu Petit ${ }^{1}$, Toyohiro Chikyow ${ }^{1}$, Kiyoto Matsuishi $^{2}$, Yutaka Wakayama ${ }^{1,(a)}$

${ }^{1}$ Advanced Electronic Materials Center, National Institute for Materials Science,

1-1 Namiki, Tsukuba, 305-0044, Japan

${ }^{2}$ Graduate School of Pure and Applied Science, University of Tsukuba,

1-1-1 Tennoudai, Tsukuba, 305-8573, Japan

(a) corresponding author: Wakayama.yutaka@nims.go.jp

Tel: +81-29-860-4403 / Fax: +81-29-860-4916 


\section{Abstract}

A molecular superlattice consisting of alternate layers of N,N'-dioctyl-3,4,9,10-perylenedicarboximide (PTCDI-C 8 ) and quaterrylene was prepared by using an ultra-slow deposition technique. Film growth under equilibrium conditions with precise optimization of the substrate temperature enabled the layer-by-layer stacking of hetero-molecules at a single-layer level. The morphology of the films and the orientation of the molecules in each layer were analyzed by atomic force microscopy (AFM) and an x-ray reflection (XRR) technique.

Keywords: oganic semiconductor, superlattice, x-ray reflectometry, ultra-slow deposition

PACS: 61.05.cm, 68.35.bm, 68.35.Ct, 68.60.Dv, 68.65.Cd 
The main purpose of this study is to establish a fine process for growing a molecular superlattice, where hetero-molecular layers are deposited alternately in monolayer level. Organic thin-film devices have recently been used in commercial applications, such as organic field-effect transistors, organic light emitting diodes and organic solar cells ${ }^{1}$. In addition to these conventional devices, organic devices based on new concepts have been reported, e.g., spin injection devices ${ }^{2}$, magnetoresistance devices ${ }^{3}$ and thyristors ${ }^{4}$. These unique characteristics require delicate arrangements of multiple molecules. A typical example can be seen in a strongly correlated system of organic materials. Non-linear conduction and/or superconductivity are induced by a charge transfer between molecules that are aligned in a low-dimensional superlattice ${ }^{4 \text {, }}$ ${ }^{5}$. In this respect, a molecular superlattice has great potential for providing innovative functionalities. This makes it essential to establish a fine process for growing a molecular superlattice at a monolayer level.

In this work, we studied the growth and structural characterization of a molecular superlattice of PTCDI- $\mathrm{C}_{8}$ and quaterrylene. The molecules, which function respectively as n-type and p-type organic semiconductors, were deposited alternately by an ultra-slow deposition process called the hot-wall technique. This technique is described in detail elsewhere ${ }^{6-8}$. An advantage of our technique is that the molecular 
layers are grown under near thermal equilibrium conditions, and so, in contrast to previous studies, precise thin film growth is possible at a monolayer level ${ }^{9-14}$. As a result, p-n junctions can be formed on a single molecular scale and charge transfer between adjacent molecules can be expected.

Molecular superlattices were fabricated on Si substrates with thermally oxidized surface layers $\left(\mathrm{SiO}_{2}\right)$ in a high vacuum $\left(\sim 10^{-6} \mathrm{~Pa}\right)$. The deposition rates were less than 0.03 molecular layers (MLs) per minute. AFM images (SII SPI-4000, dynamic force mode) and XRR spectra (Bruker-AXS, D8 Discover, Cu-K $\alpha ; \lambda=0.154 \mathrm{~nm}$ ) ${ }^{15,16}$ were obtained from various films grown at different substrate temperatures with a view to optimizing the growth conditions and examining the morphology, thickness and molecular orientation. By using XRR fitting, the layer densities were estimated to be in the $1.3-1.5 \mathrm{~g} / \mathrm{cm}^{3}$ range, which is comparable to the values reported in previous studies ${ }^{17,18}$.

First, we verified the growth of the first PTCDI-C ${ }_{8}$ monolayer on the $\mathrm{SiO}_{2}$ surface. The molecular structure is shown in Fig. 1(a). Figure 1(b) shows the XRR spectrum of the PTCDI- $\mathrm{P}_{8}$ monolayer grown at an optimized substrate temperature of $140{ }^{\circ} \mathrm{C}^{8}$. The measurement was carried out immediately after the deposition. The thickness was estimated to be $2.7 \mathrm{~nm}( \pm 0.1 \mathrm{~nm})$ from the fitting curve, indicating that 
the long axis of the molecule $(2.97 \mathrm{~nm})$ was oriented vertically to the surface normal with a small tilting angle ${ }^{8,17,18}$. A schematic illustration is shown in the inset of Fig. 1(b). Figure 1(c) is an AFM image of PTCDI-C P $_{8}(0.6 \mathrm{ML})$, which clearly shows two-dimensional growth with flat terraces. The surface profile of the AFM images (a typical profile is shown in the inset) revealed that the terraces had an average height of $2.2 \mathrm{~nm}$, which is smaller than that estimated with $\mathrm{XRR}$, and involved a certain fluctuation of about $\pm 0.2 \mathrm{~nm}$. We found that the thickness (height) had a tendency to decrease with time. For example, the reduction in thickness to approximately $1.8 \mathrm{~nm}$ $( \pm 0.2 \mathrm{~nm})$ was confirmed by XRR and AFM 2 months after the deposition (not shown here). These results imply that the PTCDI-C ${ }_{8}$ monolayer on the $\mathrm{SiO}_{2}$ is unstable and the molecular orientation inclines gradually.

Next, we examined the surface morphologies, thickness and molecular orientation of quaterrylene/PTCDI-C 8 double layers (see Fig. 2(a)). The AFM images in Fig. 2(b)-(d) show the surface morphologies of the quaterrylene layers deposited on the underlying PTCDI-C P $_{8}$ monolayer. A clear dependence on the substrate temperature was observed. Quaterrylene layers tended to grow in a three-dimensional manner below $150{ }^{\circ} \mathrm{C}$. Meanwhile, the substrate temperature at $160{ }^{\circ} \mathrm{C}$ produced $2 \mathrm{D}$ layers at the monolayer level. As a result, a well-defined quaterrylene/PTCDI-C 8 
bilayer was formed. The surface profile shown in the inset proves that the quaterrylene molecules have an upright orientation, i.e., the long axis of the molecule $(1.8 \mathrm{~nm})$ is perpendicular to the $\mathrm{SiO}_{2}$ surface normal.

To examine the total thickness of the quaterrylene/PTCDI-C ${ }_{8}$ bilayer, the XRR spectrum was measured as shown in Fig. 3(a). It should be noted that the total thickness estimated with the XRR measurement was only $3.0 \mathrm{~nm}$, which was less than the sum for the two molecules $(2.8 \mathrm{~nm}+1.8 \mathrm{~nm})$. We speculate that the reduction in the total thickness can be attributed to the instability of the PTCDI- $\mathrm{C}_{8}$ layer as already mentioned. The orientation of the PTCDI- $\mathrm{C}_{8}$ layer changed during the quaterrylene deposition presumably as a result of heating the substrate. To confirm this presumption, we investigated the effect of temperature on the $\mathrm{PTCDI}^{-\mathrm{C}_{8}}$ orientation by thermally treating a PTCDI-C 8 single layer on $\mathrm{SiO}_{2}$ at $160{ }^{\circ} \mathrm{C}$ for 3 hours after the deposition. The XRR spectrum in Fig. 3 (b) reveals that the thickness was reduced to $1.2 \mathrm{~nm}$. This result means that the thermal treatment caused the molecules to lie flat as shown in the inset. Based on these analyses, we concluded that the quaterrylene/PTCDI-C 8 bilayer consisted of a vertically orientated quaterrylene layer $(1.8 \mathrm{~nm})$ on a prostrate PTCDI-C 8 layer $(1.2 \mathrm{~nm})$, which yielded a total thickness of $3.0 \mathrm{~nm}$. 
Subsequent layers were deposited and the molecular orientations of each layer were examined. The substrate temperatures were optimized to $160{ }^{\circ} \mathrm{C}$ for all the upper layers (2nd-4th layers), in a similar way to that shown in Fig. 2. AFM images were obtained to confirm the surface flatness as shown on the right in Fig. 4(a). The XRR spectra in Fig. 4(b) were measured from thus prepared PTCDI-C 8 /quaterrylene multiple layers. The fitted pattern revealed the thicknesses of the respective layers as shown in Fig. 4(b). The quaterrylene layers (2nd and 4th layers) were very stable with a constant thickness of $1.7 \mathrm{~nm}$, which is almost the same as that of the upright orientation. On the other hand, the thickness of the PTCDI-C 8 layer (3rd layer) sandwiched between quaterrylenes exhibited a certain variation: $2.3 \mathrm{~nm}$ for as-deposited and $1.8 \mathrm{~nm}$ after the $4^{\text {th }}$ layer deposition. This variation in thickness resulted from the instability of the PTCDI-C 8 layer and the thermal effect that occurred during the deposition of the other layers as discussed for the first PTCDI-C 8 layer. In the XRR spectra, we observed a disagreement between the experimental data and the fitted patterns of around 4 degrees. This is accounted for by the fact that the diffraction peak from the (001) lattice plane of PTCDI-C 8 and quaterrylene layers overlapped the reflection patterns. To illustrate this effect, the diffraction peak from the (002) plane observed around 8 degrees is shown in the inset. 
In summary, we produced a molecular superlattice of quaterrylene/PTCDI- $\mathrm{C}_{8}$ using a fine process, where we precisely optimized the deposition conditions for $2 \mathrm{D}$ layer growth. The morphologies and molecular orientations in each layer were clarified by AFM and XRR. As a result, the molecular superlattice was found to consist of inclined PTCDI- $\mathrm{C}_{8}$ and upright quaterrylene layers. The molecules in this study function as $\mathrm{n}$ - and p-type semiconductors, respectively ${ }^{19,20}$. Therefore, this successful layer by layer growth of a molecular superlattice, which has offsets at the HOMO-LUMO energy levels, opens up the possibility of carrier transfer and carrier confinement in a similar manner to that observed with conventional inorganic semiconductors. 


\section{References}

[1] M. Ichimura, S. H. Noh, T. Ishibashi, N. Ueda, and S. Tamura, SPIE Proceedings 5937 (2005), 593703.

[2] M. Ohishi, M. Shiraishi, R. Nouchi, T. Nozaki, T. Shinjo, and Y. Suzuki, Jpn. J. Appl. Phys. 46 (2007), L605.

[3] S. Sakai, K. Yakushiji, S. Mitani, K. Takanashi, H. Naramoto, P. V. Avramov, K. Narumi, V. Lavrentiev, and Y. Maeda, Appl. Phys. Lett. 89 (2006), 113118.

[4] F. Sawano, I. Terasaki, H. Mori, T. Mori, M. Watanabe, N. Ikeda, Y. Nogami, and Y. Noda, Nature 437 (2005), 522.

[5] N. Toyota, M. Lang, and J. Müller, Low-Dimensional Molecular Metals, (Springer Series in Solid-State Sciences, 154, Springer-Verlag Berlin Heidelberg, 2007)

[6] H. Sasaki, Y. Wakayama, T. Chikyow, E. Barrena, H. Dosch, and K. Kobayashi, Appl. Phys. Lett. 88 (2006), 081907.

[7] R. Hayakawa, M. Petit, Y. Wakayama, and T. Chikyow, Organic Electronics 8 (2007), 631.

[8] M. Petit, R. Hayakawa, Y. Wakayama, and T. Chikyow, J. Phys. Chem. C. 111 (2007), 12747. 
[9] F. F. So, S. R. Forrest, Y. Q. Shi, and W. H. Steier, Appl. Phys. Lett. 56 (1990), 674.

[10] A. Ishitani and T. Nonaka, Surf. Interface Anal. 21 (1994), 356.

[11] E. Kuwahara, H. Kusai, T. Nagano, T. Takayanagi, and Y. Kubozono, Chem. Phys.

Lett. 413 (2005), 379.

[12] T. Schmitz-Hübsch, F. Sellam, R. Staub, M. Törker, T. Fritz, Ch. Kübel b, K.

Müllen, K. Leo, Surf. Sci., 445 (2000), 358.

[13] F. Sellam, T. Schmitz-Hübsch, M. Toerker, S. Mannsfeld, H. Proehl, T. Fritz, K.

Leo, C. Simpson, K. Müllen, Surf. Sci., 478 (2001), 113.

[14] T. Dienel, C. Loppacher, S. C. B. Mannsfeld, R. Forker, T. Fritz, Adv. Mater., 20 (2008), 959.

[15] V. Holy, J. Kubena and I. Ohlidal, K. Lischka, and W. Plotz, Phys. Rev. B 47 (1993), 15896.

[16] XRR simulations and fitting were calculated by using DIFFRAC ${ }^{\text {plus }}$ LEPTOS software (Bruker AXS).

[17] A. L. Briseno, S. C. B. Mannsfeld, C. Reese, J. M. Hancock, Y. Xiong, S. A. Jenekhe, Z. Bao, and Y. Xia, Nano Lett., 7 (2007), 2847. 
[18] T. N. Krauss, E. Barrena, X. N. Zhang, D. G. de Oteyza, J. Major, V. Dehm, F.

Würthner, L. P. Cavalcanti, and H. Dosch, Langmuir, 24 (2008), 12742.

[19] Reid J. Chesterfield, John C. McKeen, Christopher R. Newman, Paul C. Ewbank, Demtrio, A. da Silva Filho, Jean-Luc Brdas, Larry L. Miller, Kent R. Mann, and C.

Daniel Frisbie, J. Phys. Chem. B, 108 (2004), 19281.

[20] Ryoma Hayakawa, Matthieu Petit, Yutaka Wakayama and Toyohiro Chikyow, Journal of Physical Chemistry C, 111 (2007), 18703. 


\section{Figure Captions}

Figure 1 (a) Chemical structure of PTCDI-C ${ }_{8}$ molecule. (b) XRR spectrum of PTCDI-C 8 monolayer. The experimental result (red circles) and fitted line (blue line) revealed a layer thickness of $2.8 \mathrm{~nm}$. The molecular orientation is shown in the inset. (c) $5 \times 5 \mu \mathrm{m}^{2}$ AFM image of $0.6 \mathrm{ML}$ PTCDI-C 8 . The surface profile shows the PTCDI- $\mathrm{C}_{8}$ layer is $2.2 \mathrm{~nm}$ thick, suggesting the layer undergoes a gradual change in orientation.

Figure 2 (a) Chemical structure of quaterrylene molecule. (b)-(d) $5 \times 5 \mu \mathrm{m}^{2}$ AFM images of quaterrylene layers grown on the PTCDI-C ${ }_{8}$ monolayer at various substrate temperatures. The optimum substrate temperature for growing a $2 \mathrm{D}$ layer was $160{ }^{\circ} \mathrm{C}$.

Figure 3 (a) XRR spectrum of a PTCDI-C 8 /quaterrylene double layer. The experimental result (red circles) and fitted line (blue line) revealed a total thickness of $3.0 \mathrm{~nm}$. (b) XRR spectrum of the PTCDI-C 8 monolayer after thermal treatment, which indicates a change in molecular orientation (see Fig. 1 (b)). 
Figure 4 (a) Illustration of PTCDI- $\mathrm{C}_{8} /$ quaterrylene superlattice and AFM images of respective layers. The flat surfaces of each layer confirm well-defined 2D growth. (b) XRR spectra of PTCDI-C 8 /quaterrylene superlattice. The film constitution and the thicknesses of each layer are shown in the illustration. The disagreement between the experimental results (red circles) and fitted lines (blue lines) around 4 degrees is attributed to the diffraction peak from the (001) lattice plane. The peak from the (002) plane observed around 8 degrees is shown in the inset as a reference. 

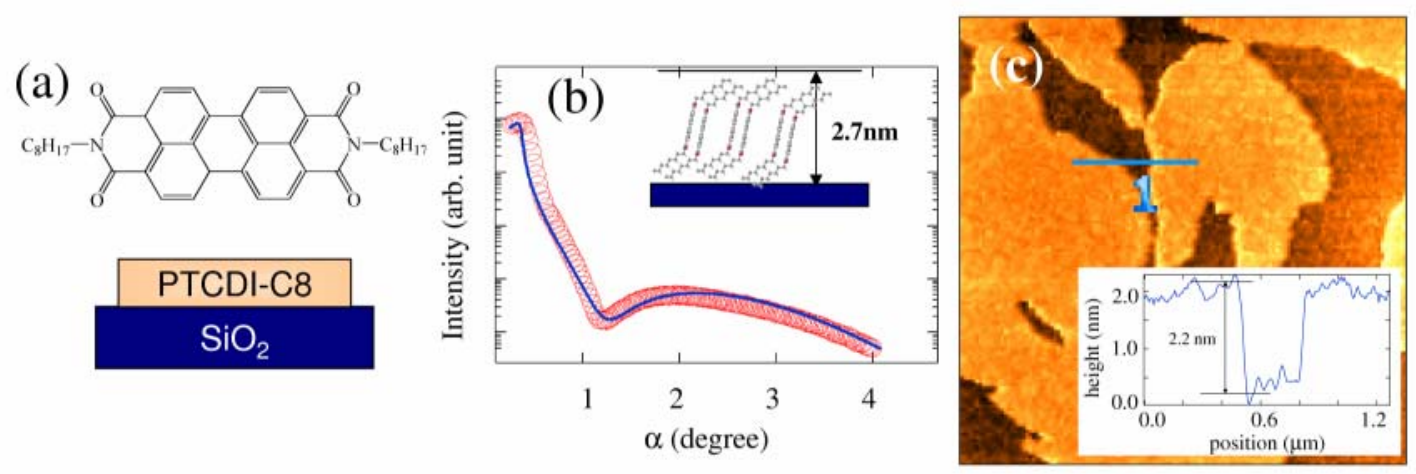

Figure1

(a)
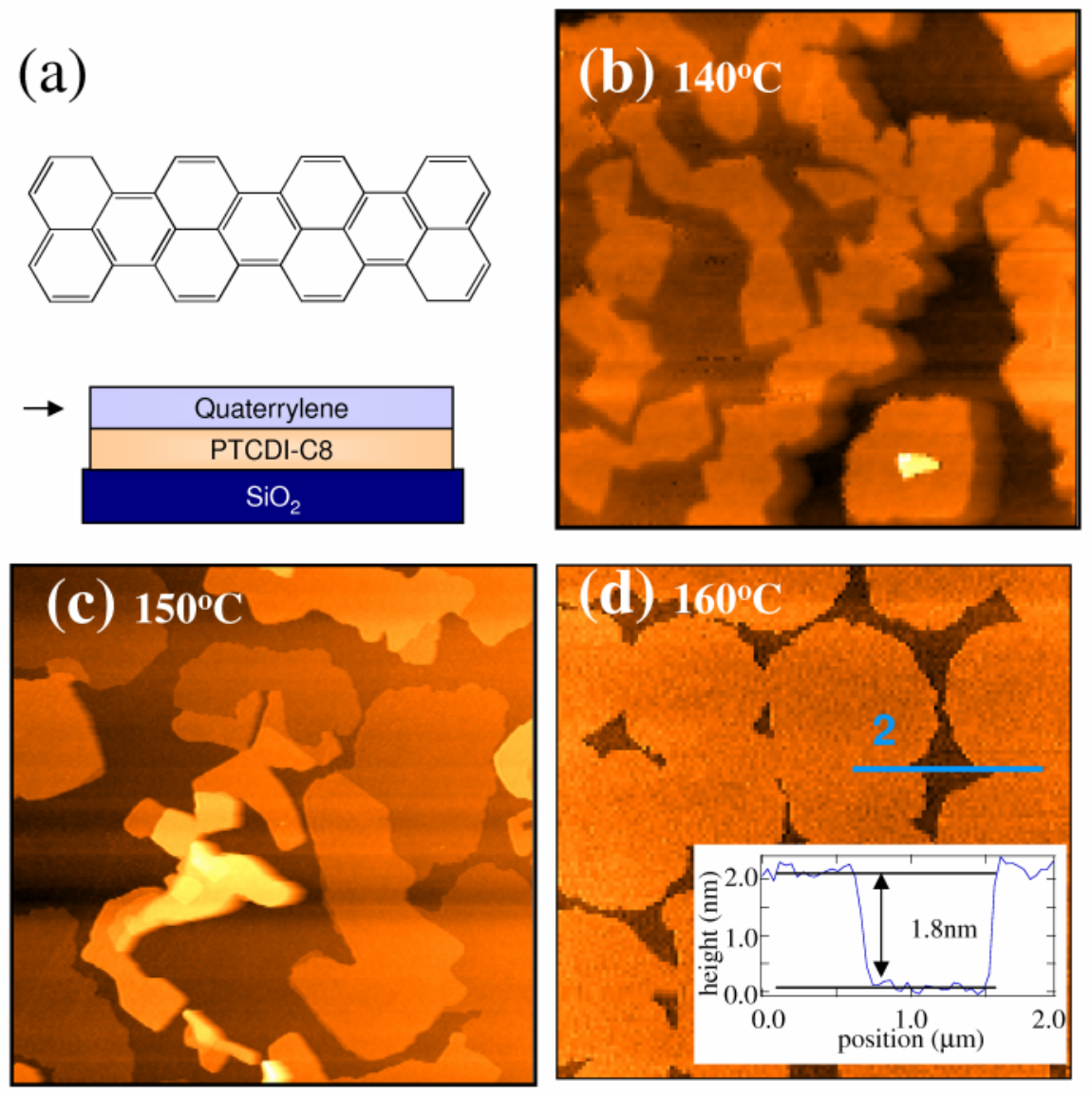

Figure2 

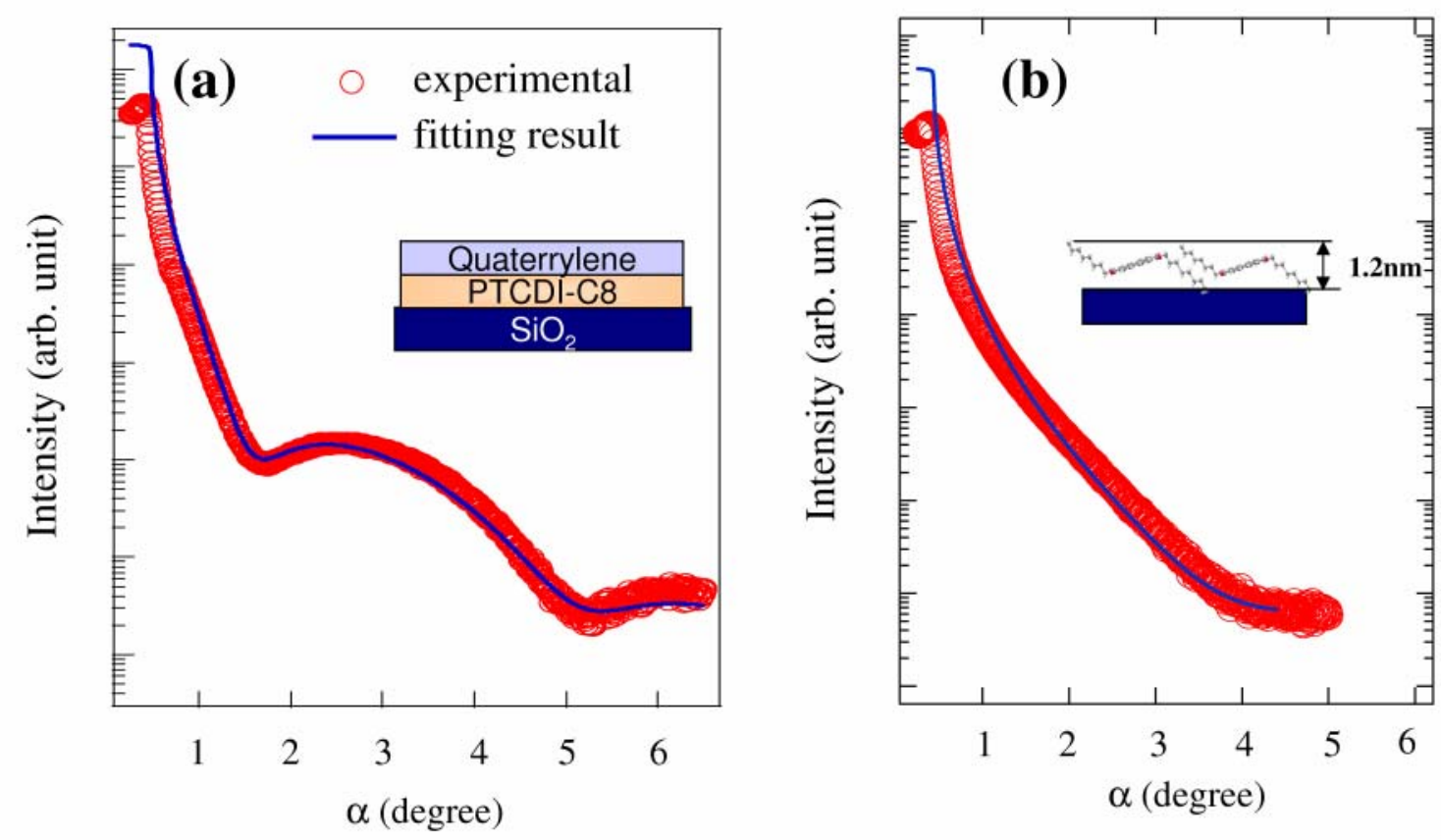

Figure3 
(a)
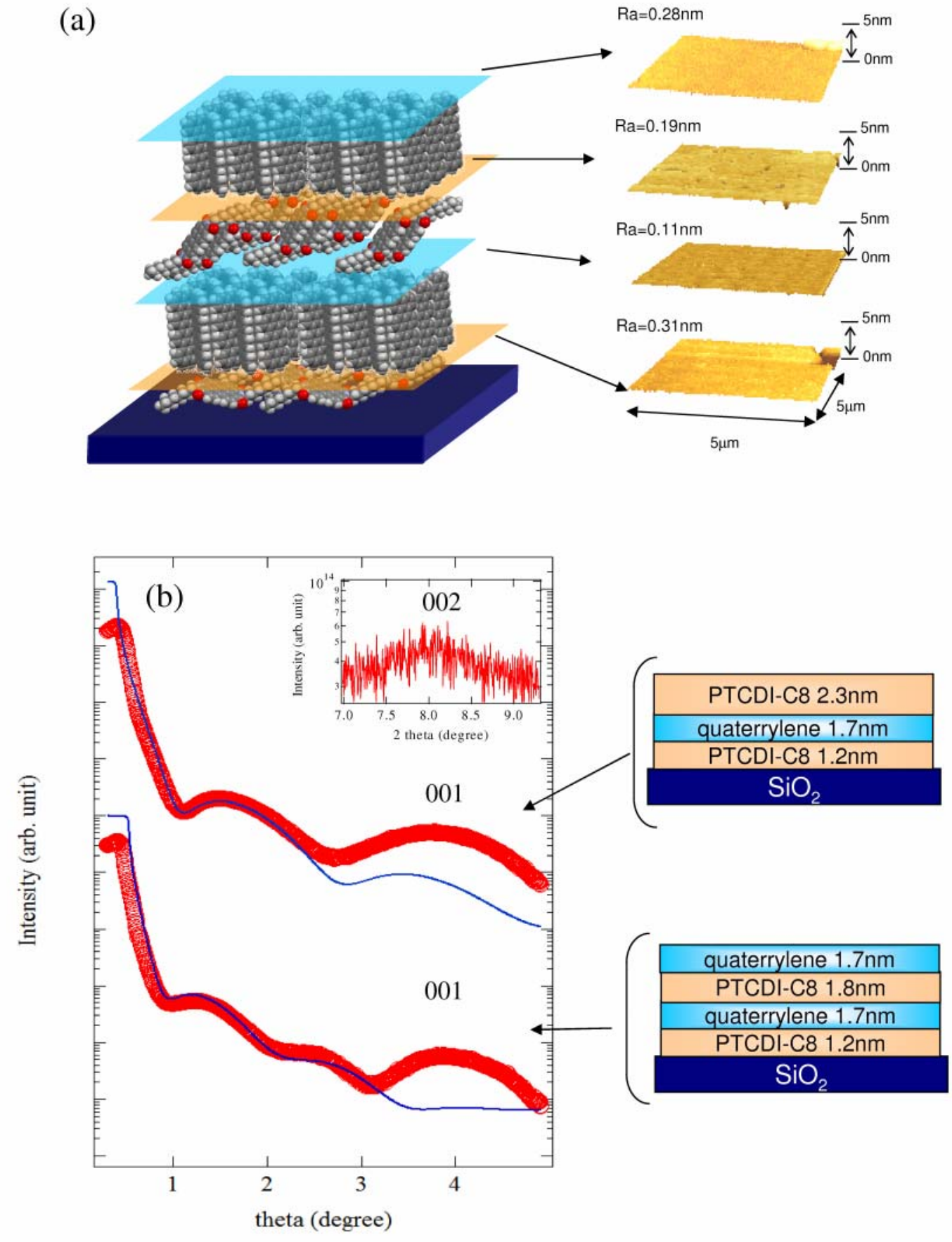

Figure4 